\title{
DRL behavior in gerbils and hamsters of both sexes
}

\author{
WILLIAM W. BEATTY \\ North Dakota State University, Fargo, North Dakota 58102
}

\begin{abstract}
Female rats acquire efficient DRL responding more rapidly than do males. In the present study, acquisition of DRL-20 behavior was studied in hamsters and gerbils of both sexes. There was no sex difference in DRL performance in gerbils, and male hamsters performed somewhat more efficiently than did females.
\end{abstract}

Previous research has indicated that sexual dimorphisms in nonreproductive behavior are frequently species dependent. For example, in most mammalian species the male is generally more aggressive than the female, but the hamster is an exception to this rule. Female hamsters have consistently been observed to be more aggressive than males (see Quadagno, Briscoe, \& Quadágno, 1977).

Species differences in other sexually dimorphic measures have also been described. Hamsters do not display the sexually dimorphic patterns of bodyweight regulation observed in the rat, but both species have similar patterns of sex differences in taste preferences (Zucker, Wade, \& Zeigler, 1972). Neither gerbils nor hamsters exhibit sex differences in pain sensitivity or avoidance behavior of the same sort displayed by the rat (Ashe \& McCain, 1972; Beatty, 1977; Beatty \& Fessler, 1977; Beatty \& Holzer, in press). In the present experiment DRL behavior was studied in hamsters and gerbils. In earlier studies with rats (e.g., Beatty, 1973, 1977; Kearley, van Hartesveldt, \& Woodruff, 1974), females consistently acquired efficient DRL-20 behavior more rapidly than did males.

\section{METHOD}

\section{Subjects}

The subjects were 16 male and 16 female Syrian hamsters (Mesocricetus auratus) obtained from ARS-Sprague Dawley, Madison, Wisconsin and 16 male and 16 female Mongolian gerbils (Meriones unguiculatus) (MON-TUM) obtained from Tumblebrook Farms, West Brookfield, Massachusetts. The animals were about 10 weeks old when they arrived in the laboratory; for the next 2 weeks they were adapted to the $12: 12$ light-dark cycle in effect throughout the experiment. They were caged singly in an air-conditioned animal room that was illuminated from $0900-2100 \mathrm{~h}$. The subjects had free access to water and sufficient food in the home cage to maintain body weight at approximately $85 \%$ of the free-feeding level established during the 2-week period in which the animals were adapted to the laboratory.

Supported by Grant HD 08206 from NIH. I thank Verlin Hinsz and Bruce O'Gara, who assisted in teaching the animals.

\section{Apparatus}

Tests were conducted in conventional Gerbrands rat operant conditioning chambers equipped with feeders that dispensed 20 -mg Noyes pellets. The chambers were modified to make them more suitable for hamsters and gerbils by (1) inserting a floor made of hardware cloth on top of the standard grid bars to permit the animals to reach the response lever more easily and (2) cementing a small piece of clear plastic to the response lever so that the animals could depress it with less effort.

\section{Procedure}

The procedure was identical to that employed in previous studies of DRL behavior in rats in our laboratory (e.g., Beatty, 1973). Tests were conducted during the daylight portion of the day-night cycle since, in rats, the sex difference in DRL behavior is not observed at night (Beatty, 1977). In brief, the animals were first trained to barpress for food $(20-\mathrm{mg}$ Noyes pellets, standard formula) and given one or two additional sessions during which the animal could earn as many as 50 pellets, available on CRF. On the following day the reinforcement schedule was shifted to DRL-20. This reinforcement schedule remained in effect for 40 daily sessions, each $30 \mathrm{~min}$ long.

\section{RESULTS AND DISCUSSION}

Table 1 summarizes the DRL behavior of hamsters and gerbils in terms of efficiency scores (reinforcement/ responses). Data from an earlier study with rats (Beatty, 1977) are also shown to facilitate comparison of sex differences across species. As is evident from the table, the pattern of sex differences in DRL performance was quite different for these three rodent species. Female rats acquired efficient DRL behavior more rapidly than did males, but a similar sexual dimorphism was not observed in gerbils or hamsters. Statistical analysis of the data from gerbils revealed neither a main effect of sex nor a Sex by Blocks interaction (both Fs $<2$ ). Analysis of the data from hamsters also revealed no main effect of sex $(F<1)$, but the Sex by Blocks interaction was marginally significant $[F(7,210)=2.60, p<.05]$. The interaction arose because male hamsters obtained somewhat higher efficiency scores than did females on the latter test blocks.

The DRL behavior of hamsters and gerbils also differed from that of rats in other respects. Both hamsters and, especially, gerbils attained higher efficiency scores than did rats early in training, but showed smaller 
Table 1

Mean Efficiency $\times 100$

\begin{tabular}{lrccccccc}
\hline & \multicolumn{7}{c}{ Blocks of Five Sessions } \\
\cline { 2 - 9 } & 1 & 2 & \multicolumn{1}{c}{ 1 } & \multicolumn{1}{c}{4} & 5 & 6 & 7 & 8 \\
\hline Male Rats & 5.6 & 8.3 & 17.6 & 28.9 & 31.6 & 37.9 & 40.0 & 41.1 \\
Female Rats & 8.4 & 14.9 & 26.2 & 36.8 & 45.9 & 52.5 & 59.8 & 61.2 \\
Male Gerbils & 25.6 & 29.7 & 35.4 & 42.5 & 43.5 & 51.2 & 50.4 & 52.7 \\
Female Gerbils & 28.3 & 39.6 & 45.6 & 49.5 & 48.0 & 50.8 & 54.2 & 50.6 \\
Male Hamsters & 13.6 & 15.1 & 16.5 & 20.9 & 21.7 & 24.8 & 29.2 & 32.7 \\
Female Hamsters & 16.1 & 15.7 & 18.1 & 21.1 & 22.7 & 19.7 & 21.2 & 23.4 \\
\hline
\end{tabular}

increases in efficiency as training improved. The major reason for this differential improvement in efficiency with repeated testing for the three species was the differential change in response output with testing. While daily response totals for rats declined from about 225 250 responses/session at the outset of training to 90-125 responses/session by the end of training, responding by gerbils and hamsters remained fairly constant at about 85-110 responses/session throughout training. All three species earned more reinforcements per session as training progressed, and the magnitude of this increase was somewhat greater for rats.

It would be foolish to speculate about the origin of the species differences in DRL behavior described here since we have no strong reason to believe that our attempts to equate deprivation conditions, rates of satiation, and ease of execution of the barpress response were successful. However, it is not obvious why any failure to equate factors such as these across the three species would result in a sex difference in one species but not in the other two. Hence, the present findings with DRL behavior concur with recent studies of sex differences in other nonreproductive behaviors. With regularity, which is perhaps disconcerting, these studies reveal that sexual dimorphisms in behavior are highly dependent on the species and the situation.

\section{REFERENCES}

Ashe, V. M., \& McCain, G. Comparison of one-way and shuttleavoidance performance of gerbils and rats. Journal of Comparative and Physiological Psychology, 1972, 80, 293-296.

BEATTY, W. W. Effects of gonadectomy on sex differences in DRL behavior. Physiology and Behavior, 1973, 10, 177-178.

BEATTY, W. W. Sex differences in DRL and active avoidance behaviors in the rat depend upon the day-night cycle. Bulletin of the Psychonomic Society, 1977, 10, 95-97.

Beatty, W. W., \& Fessler, R. G. Sex differences in sensitivity to electric shock in rats and hamsters. Bulletin of the Psychonomic Society, 1977, 10, 189-190.

Beatty, W. W., \& Holzer, G. A. Sex differences in shock thresholds in rats and gerbils and the day-night cycle. Bulletin of the Psychonomic Society, in press.

Kearley, R. C.. van Hartesveldt, C., \& Woodruff, M. L. Behavioral and hormonal effects of hippocampal lesions in male and female rats. Physiological Psychology, 1974, 2, 187-196.

Quadagno, D. M., Briscoe, R., \& Quadagno, J. S. Effect of perinatal gonadal hormones on selected nonsexual behavior patterns: A critical assessment of the nonhuman and human literature. Psychological Bulletin, 1977, 84, 62-80.

Zucker, I., WAdE, G. N., \& Ziegler, R. Sexual and hormonal influences on eating, taste preferences, and body weight of hamsters. Physiology and Behavior, 1972, 8, 101-111.

(Received for publication September 26, 1977.) 